\title{
ROBUSTNESS OF CYCLIC SCHEDULES FOR THE CHARGING OF BATTERIES
}

\author{
SIMON DUNSTALL ${ }^{\oplus 1}$ and GRAHAM MILLS ${ }^{2}$
}

(Received 31 August, 2004; revised 10 October, 2006)

\begin{abstract}
In 2002 the Mathematics in Industry Study Group (MISG) investigated the question of optimally scheduling cyclic production in a battery charging and finishing facility. The facility produces various types of battery and the scheduling objective is to maximize battery throughout subject to achieving a pre-specified product-mix. In this paper we investigate the robustness of such schedules using simulation experiments that span multiple production cycles. We simulate random variations (delays) in battery charging time and find that an optimal off-line schedule yields higher throughput in comparison to a common on-line dispatching rule. This result has been found to hold for a range of expected chargingtime delays and has significant practical implications for scheduling battery charging and finishing facilities.
\end{abstract}

2000 Mathematics subject classification: primary 90B35; secondary 90B30.

Keywords and phrases: scheduling, simulation, robustness.

\section{Introduction}

Exide Technologies operates a manufacturing facility in South Australia that in 2002 . produced about two million lead-acid batteries per annum. The facility was one of the topics investigated at MISG 2002. In this paper we study an abstracted model of the battery charging and finishing operations of this facility, where finishing refers to activities such as battery cleaning and labelling. The model and a mixed-integer mathematical programming formulation for the associated scheduling problem is described in Dunstall and Mills [2].

The facility produces a range of batteries and these batteries can be formed into 21 battery-types (which we will refer to as products), each of which has a minimum charge

\footnotetext{
${ }^{1}$ CSIRO Mathematical and Information Sciences, Private Bag 33, Clayton South 3169, Australia; e-mail: Simon.Dunstall@csiro.au.

${ }^{2}$ CSIRO Mathematical and Information Sciences, Private Bag 2, Glen Osmond 5064, Australia; e-mail: Graham.Mills@bigpond.com.

(C) Australian Mathematical Society 2007, Serial-fee code 1446-181 1/07
} 
duration and a known weekly demand. The minimum charge duration is constant for each product and is between 18 hours and 63 hours. In practice the charge duration is subject to variations (disruptions) that are caused by ambient temperature fluctuations and other environmental effects that can inhibit heat transfer from batteries that are being charged. These largely unpredictable effects lead to charge durations that are greater than the minimum charge durations.

From the demand information we can compute a target product mix, which we define as the desired proportion of batteries of each product relative to the total number of batteries produced in a week. Weekly production can be characterized in terms of the production ratio for each product. For a given product the production ratio is defined as the ratio between the number of batteries of this product that are produced and the demand for this product. When scheduling the facility we seek to maximize the throughput of the facility subject to maximizing the minimum production ratio.

The charging and finishing operations are carried out on batteries that are arranged on stills (or racks) which hold a certain number of individual batteries, all of which must be of the same product. The number of batteries in a still is fixed for each product and we can express the product mix, production ratios, and the charging and finishing schedules in terms of stills. The number of stills is the system is constant and can often be a binding constraint when scheduling.

The charging area is comprised of a fixed number of bays and operates continuously. Each bay can hold one still of any product and must charge batteries non-preemptively. The charging process is automated and the supervisory demands on human operators can be neglected. However, loading and unloading of stills does require human intervention and of particular concern is the loading-rate which is dependent on the time-of-week and can limit battery throughput. The activities undertaken in the finishing area are predominantly manual in nature. The finishing area does not operate continuously and can be approximated by a classical parallel machine model (see, for example, Lawler et al. [5]) with unit-length operations, constant processing rates and machine unavailability periods. Overall, the limiting factors when scheduling the facility are the number of stills, the number of charging bays, the charging-area loading rate and the throughput capacity of the finishing area.

The purpose of this paper is to compare and contrast three straightforward scheduling strategies under varying degrees of charge-duration disruption. Each of these scheduling strategies is cyclic in nature: that is, a weekly schedule is formed and the facility cycles through this schedule over a period of many weeks. In all cases the weekly schedule is determined using the minimum charge durations (that is, we adopt a naive assumption that disruptions are entirely unpredictable). In the first strategy ("MIP") we find the optimal schedule through application of the MIP formulation. In the second strategy ('SPT") we sequence stills according to non-decreasing charge time: this is equivalent to the well-known shortest processing time (SPT) rule. In the 
third strategy ("RND") we generate a random sequence of stills. The third strategy is a useful point of comparison between the other two strategies, although it is interesting to evaluate the robustness of RND strategy under charge-time variation.

It is highly relevant to observe that, in the context of this scheduling problem, offline (or "proactive") SPT sequencing is indistinguishable from on-line (or "reactive") SPT dispatching of stills. This is because, in comparison to off-line SPT sequencing, no additional information will be used for on-line SPT dispatching. However, in a more sophisticated on-line strategy one could make use of dynamic state information such as the current expected finishing time of charging operations or the number of stills queueing in front of the finishing area. Because SPT sequencing represents a very simple on-line scheduling strategy, by comparing the performance of the MIP and SPT schedules, we gain insight into the performance difference between simple online and optimal off-line schedules and into the possible scope for improving dynamic schedule performance using sophisticated on-line scheduling strategies.

The papers by Turnquist [9] and Dunstall and Mills [2] address the issue of production scheduling in multi-product battery charging and finishing facilities. In both papers the various battery models can be grouped into generic battery types, and a generic type becomes a particular battery model (or product) only at the last stage (finishing stage) of the production process. In this paper we refer to a generic type as a product and do not account for particular battery models other than by scheduling the finishing operations for each product.

Turnquist describes the application of a network flow algorithm to schedule multiproduct battery plants on a month-by-month basis over a yearly horizon. Each plant produces a subset of a particular firm's several hundred battery models and the allocation of models to plants is a higher-level decision that precedes production scheduling. The overall goal in the Turnquist model is to find a minimum-cost solution where cost is dependent on inventory levels and on planned production overtime. The capacity limitations of the facility act to constrain the schedule on a monthly basis.

Dunstall and Mills consider battery production at a finer level of detail. They schedule the use of stills, charging bays and battery finishing lines for each of 42 periods in a (cyclic) working week, and use a mixed-integer programming formulation to solve the scheduling problem. In this paper we make use of this formulation after making some minor revisions (see Section 2.1). Our scheduling problem and modelling approach is somewhat similar in spirit to that of Panton and Beaumont [8]. Panton and Beaumont considered the scheduling of autoclaves (as batch processors) into which components made of composite materials were placed for curing. For this process the components were laid-up on tools which were mounted on trolleys, both the tools and trolleys being limited resources.

We are interested in evaluating the performance of the MIP, SPT and RND scheduling strategies under varying degrees of charge-duration perturbation. The schedules 
that are produced by the strategies in question are not affected by the degree of perturbation. Thus the scheduling strategies will be evaluated according to their ability to produce schedules that are robust relative to unpredictable events, in a scenario where schedules cannot be changed as a result of these events. This scenario can be compared to those in which rescheduling occurs (see, for example, Vieira et al. [10]) and robustness in that case can be assessed through measures of rescheduling stability and scheduling nervousness (see, for example, Gan [3]).

Our scheduling strategy performance evaluation is based on using a discrete event simulation model of the battery assembly, charging and finishing areas. The simulation has been carried out using the ARENA software package [1]. We adopt average weekly production as our main scheduling performance measure. In the simulation we use one long (that is, many-cycle) replication and determine the variance by batch means.

Mignon, Honkomp and Reklaitis [7] use a similar methodology to investigate schedule robustness under uncertainty. In their experiments they adopted a performance measure that was common to the scheduling and simulation parts of the study. For a performance measure $p$ they use a Robustness $R$ and Performance $P$ defined by

$$
R=100-\left(\frac{100 \sigma(p)}{\bar{p}}\right) \text { and } P=\frac{100 \hat{p}}{\bar{p}},
$$

where $\bar{p}$ is the value of $p$ in the deterministic model, $\hat{p}$ is the average value and $\sigma(p)$ is the standard deviation of $p$ over the replications. They model stochastic processing times as a normal distribution with a mean of one and a standard deviation of one tenth. They average their performance measure over 500 replications. They compare the optimization schedule with a schedule that advances jobs if there is slack and a schedule that plans for lateness by advancing the due dates. They find that the third strategy has the best performance. In a second paper Honkomp, Mockus and Reklaitis [4] consider variation in processing times and equipment disruptions. They compare the optimized schedule from a deterministic mixed-integer programming model with the simulation of rescheduling methods when stochastic events occur. These experiments, as well as those of Liu [6], deal with due-date constrained jobs and so the results (for example, Liu finds that operations-based strategies are better than job-based strategies in flow shops) are of interest but not directly applicable to the studies in this paper.

\section{Solution methods}

We developed a trace-driven discrete event simulation model in which the base schedule is used to sequence jobs through a charging and finishing area. The loading and finishing resources for processing the jobs are made available on a shift basis by 
restricting the resources to a five day per week operation. The charging area operates 24 hours per day throughout the week.

A schedule is used as input to the simulation run and it specifies the (cyclic) sequence of products to be released into the system on stills. The simulation follows this sequence without deviation. The batteries for the first still in the sequence cannot be released until the last still of the previous cycle has been loaded, and a still cannot be loaded prior to the beginning of the week that corresponds to this repetition of the cycle. For this reason the simulation will produce batteries in the correct product mix because the schedules are constrained to have the correct product mix.

The differences in minimum charge times and the unplanned variations in process times will affect the order in which batteries complete charging according to the simulation. However, once batteries are charged, all stills require the same amount of work input to complete. Thus, at least in the simulation, loaded stills will complete a circuit of the system in approximately the same order as they begin it. We stop releasing new still-loads of batteries whenever there is a queue of such loads for stills.

The stills queue for a space in the charge area that is restricted to 53 spaces. On completion of their charge duration they leave the charge area and enter the buffer ahead of the finishing line. This line operates for two shifts with capacity to finish 30 and 20 stills per shift, respectively, on weekdays. Stills are released for reuse once finishing is completed.

We tested three strategies (MIP, SPT and RND) for proactive scheduling and in all the scenarios we replaced the average deterministic charge times $w$ by Norm $(w(1+$ $k \mu), \sigma \sqrt{k w}$ ), where $\mu$ is the mean and $\sigma$ is the standard deviation of an individual disruption and $k$ is the disruption frequency. We reason that jobs with longer charge time are more likely to have more disruptions. We used values of 0.5 and 0.1 for $\mu$ and $\sigma$, respectively. For each schedule the jobs have their processing time modified by the normal distribution when they enter the charging area.

The simulation models were run for one replication representing a duration of 1018 weeks. The first two weeks were used as a warmup period to fill the production line with stills from an initially empty system. Variability in the charge times was introduced from the second week. Thus the simulation starts to gather statistics at the beginning of the third week (the number of jobs released, the number of stills finished and so on) and we continue to gather statistics for the remaining 1015 weeks.

2.1. Offline schedules To generate optimal off-line schedules we adopt the mixedinteger programming formulation first introduced in [2]. This MIP has variables which keep track of the number of stills that are assembled, queueing for the charger, in the charging area, queueing for finishing, and finished. We set the initial and ending boundary conditions to give a wrap-around (cyclic) steady-state production schedule with a one-week cycle duration. 
We introduce some notation and parameters:

$p$ products;

$D_{p}$ sales demand;

$T$ number of time periods (42 unless otherwise stated);

$t$ periods $1,2,3, \ldots, T$;

$R$ number of charging-area spaces (53 unless otherwise stated);

$I$ available number of stills (65 unless otherwise stated);

$L_{t}$ maximum number of stills able to be loaded in period $t$;

$S_{p t}$ period $t^{\prime}$ is a member of $S_{p t}$ if stills of product $p$ loaded in period $t^{\prime}$ remain on charge in period $t$;

$w_{p}$ charge time for product $p$ (spring, in periods);

$F_{t}$ an indicator of the number of finishing lines working in period $t$;

FCAP maximum finishing capacity (per shift) of a finishing line;

$\epsilon$ a small factor to break degeneracy in the solutions.

We introduce some variables:

$a_{t}$ number of stills assembled in period $t$;

$q_{t}$ the inventory of assembled stills (of any product) at the end of period $t$;

$x_{p t}$ number of stills of product $p$ that start charging in period $t$;

$y_{p t}$ number of stills of product $p$ that finish charging in period $t$;

$z_{t}$ number of stills processed on the finishing line in period $t$;

$c_{t}$ number of stills in the charging area in period $t$;

$b_{t}$ number of stills in the buffer at the end of period $t$;

$f$ fraction of the finishing capacity utilized;

$r$ minimum value of the production to demand ratio for any product.

The aim is to maximize production while maintaining the specified product mix. Hence in the formulation we maximize the minimum production ratio $r$ over all products: we sacrifice over-production of one product in favour of increasing the production of an alternative product that may be under-produced (relative to the demand). Thus the model aims to maintain the product mix rather than produce one type of battery at the expense of another. We state the problem as follows

$$
\text { Maximize } 1000 r+\epsilon \sum_{t} a_{t}-q_{0}
$$

subject to

$$
\begin{aligned}
a_{t} & \leq L_{t} \quad \text { for all } t, \\
q_{t} & =q_{t-1}+a_{t}-\sum_{p} x_{p t} \text { for all } t, \\
c_{t} & =\sum_{p} \sum_{s \in S_{p t}} x_{p s} \text { for all } t,
\end{aligned}
$$




$$
\begin{aligned}
& c_{t} \leq R \quad \text { for all } t, \\
& x_{p, t}=y_{p, t+w_{p}} \text { for all } p \text { and } t: t+w_{p} \leq T, \\
& x_{p, t}=y_{p, t+w_{p}-T} \text { for all } p \text { and } t: t+w_{p}>T, \\
& z_{t}=\sum_{p} y_{p t}+b_{t-1}-b_{t} \text { for all } t, \\
& z_{t} \leq f . F_{t} \text { for all } t, \\
& f \leq \mathrm{FCAP}, \\
& q_{t}+c_{t}+b_{t} \leq I \text { for all } t, \\
& \sum_{t} \frac{x_{p t}}{D_{p}} \geq r \text { for all } p .
\end{aligned}
$$

The objective function (2.1) and constraint (2.12) enable the maximization of the minimum production ratio amongst the set of products. The second term in the objective function has a marginal effect designed to break degeneracy in the solution space, but can result in over-production. The third term ensures that the inventory of assembled stills is kept at a minimum and thus it also acts to eliminate degenerate solutions.

Constraints (2.2) reflect the limited loading capacity of the assembly line. We used a loading capacity of 30 stills per shift for the day and afternoon shift and 5 stills for the night shift.

Constraints (2.3) are conservation constraints relating to flow of stills into and out of the buffer between the loader and the charging area.

Constraints (2.4) determine the number of stills in the charging area during period $t$. This is calculated by summing the number of stills started in each of the periods for which such stills would remain under charge in period $t$. Constraints (2.5) limit the number of stills in the charging area to be no more than the number of spaces available.

Constraints (2.8) are conservation constraints relating to flow of stills into and out of the buffer between the charging area and the finishing lines. The number of stills coming off charge during period $t$ plus the number of stills in the buffer at end of the preceding period (period $t-1$ ) must be equal to the number of stills finished plus the number of stills in the buffer at the end of period $t$. We take the value of $b_{0}$ to be equal to the value of $b_{T}$.

Constraints (2.6) and (2.7) ensure that stills which start charging in period $t$ are set to finish charging in period $t+w_{p}$ (or period $t+w_{p}-T$ if $t+w_{p}>T$ ).

Constraints (2.9) limit the finishing of stills to the capacity of the finishing lines during working shifts. Finally, constraint (2.10) limits the throughput of the finishing lines to be no more than their maximum capacity.

Constraints (2.11) limit the total number of stills in use in the system.

To generate SPT and RND off-line schedules we simply sort the 239 stills (the 


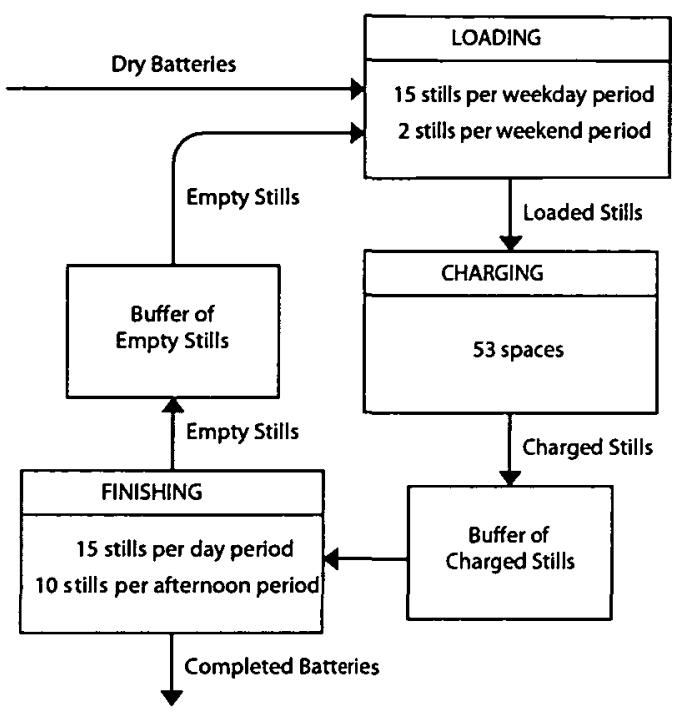

FIGURE 1. Process diagram for assembling, charging and finishing batteries.

weekly production) in non-decreasing and random minimum-charge-time order, respectively. For our simulation it is the order of still-assembly that is important and so a sequence of stills is sufficient. The shortest processing time model uses hours as the units of measurement.

\section{Computational experiments}

Our model of the battery production facility is based on a real-world case study. Each battery is assembled, charged and finished in the facility. Figure 1 is a schematic of the production process.

The assembly area either produces dry batteries that can be stored in pallets prior to charging, or acid filled batteries that are held in stills for transfer to the charging spaces when a free space becomes available. Each (identical) still holds between 60 and 96 batteries, depending on the product. A loaded still is always fully loaded with a single product. We decided to use the number of stills of each product as the production unit rather than the number of batteries. Likewise we have simplified thi problem by selecting half-shifts (four hours) as the time period.

Table 1 lists the product groups, the total charge time for the spring season in hours and periods, and the number of weekly stills required for average daily demand for each product for a product mix of 22500 batteries per week. According to these 
TABLE 1. Data on product type, spring season average charge times in hours and periods, and weekly demand in stills.

\begin{tabular}{|l|c|c|c|c|c|c|c|c|c|c|c|c|c|c|c|c|c|c|c|c|c|}
\hline Product Type & A & B & C & D & E & F & G & H & I & J & K & L & M & N & O & P & Q & R & S & T & U \\
\hline Charge Hours & 63 & 60 & 64 & 55 & 49 & 47 & 55 & 60 & 56 & 56 & 54 & 62 & 19 & 26 & 22 & 28 & 20 & 26 & 20 & 23 & 18 \\
\hline Charge Periods & 16 & 15 & 16 & 14 & 13 & 12 & 14 & 15 & 14 & 15 & 14 & 16 & 5 & 7 & 6 & 7 & 5 & 7 & 5 & 6 & 5 \\
\hline Weekly Stills & 28 & 2 & 5 & 11 & 2 & 1 & 5 & 8 & 8 & 8 & 2 & 6 & 20 & 23 & 7 & 3 & 35 & 1 & 25 & 34 & 5 \\
\hline
\end{tabular}

figures, the number of stills to be produced is 239 weekly, or about 48 daily (Monday to Friday). The data contained in Table 1, relating to the set of products and their average charging times in spring, has been used throughout.

Our problem can be viewed as a production system where the finishing area is equivalent to a single machine operating at a constant rate, and the charging area is a batch processor with a limited input rate (the loading rate). We are potentially constrained in our scheduling decisions by both the capacity of these processors as well as the resource of stills.

We use the following capacity assumptions regarding factory operations.

(1) There are 3 shifts ( 8 hours each) every day, and they start at 6am (morning shift), $2 \mathrm{pm}$ (afternoon shift) and 10pm (night shift).

(2) There are 65 stills in the factory.

(3) Loading can be done at a rate of up to 30 stills during morning and afternoon shifts, that is, 15 stills per half-shift or period.

(4) Up to 4 stills can be loaded on night or weekend shifts, that is, 2 stills per period.

(5) There are 53 spaces (that is, for 53 stills) in the charging area. The charging area can operate for 7 days a week.

(6) Finishing can be done at a rate of up to 30 stills during morning shifts and up to 20 stills during afternoon shifts, weekdays, that is, 15 stills in a morning period and 10 stills in an afternoon period.

(7) No finishing is done during the weekends and at nights.

In practice there are a number of controls available to production planners that can be used to affect the production schedule. For example, they can change the production sequence for products or use overtime shifts for assembly and finishing if required. In this paper we manipulate the production sequence and treat all other factors as fixed. Finally, we note that a schedule that minimizes the total duration of idle time for finishing area will be optimal with respect to facility throughput.

Results were for each of the three scheduling strategies under variation in the disruption ratio $k$. The following performance statistics have been gathered during our simulation runs:

- $\mathrm{MxW}$ is the maximum number of stills busy during the week,

- AvW is the average number of stills busy during the week, 
- MxQ is the maximum number of stills in the queue ahead of the charger at the end of a period,

- AvQ is the average number of stills in the queue ahead of the charger at the end of a period,

- $\mathrm{MnC}$ is the minimum number of stills charging during the week,

- $\mathrm{AvC}$ is the average number of still charging during the week,

- $\mathrm{MxB}$ is the maximum number of stills in the buffer at the end of a period,

- AvB is the average number of stills in the buffer at the end of a period,

- AvP is the average weekly production over the simulation

- HfP is the half-width of the $95 \%$ confidence interval of the weekly production.

$\mathrm{MxW}$ and $\mathrm{AvW}$ relate to the work in progress as they measure the number of stills assembled, in the queue, in the charging and in the buffer areas. The facility throughput objective, that is, primary performance measure, is AvP.

In the simulation the start of the first period corresponds to the start of the morning shift on Monday. The base instance of the model has a loading capacity of 344 stills, spaces for charging 53 stills, availability of 65 stills, finishing capacity for 250 stills in normal work hours and a weekly production demand for 239 stills.

Table 2 gives performance statistics for the optimal off-line schedule execution without disruptions to charge-durations (that is, with minimum charge-durations). These statistics are computed using the values assigned to the MIP decision-variables. The schedule permits the charging of 241 stills per week in 58 batches, where a batch is a contiguous sub-sequence of stills of the same product. The demand for each product is met and there is an overproduction of two units relative to the total demand of 239 stills.

TABLE 2. Performance measures for the planning model with 53 spaces, 65 stills and finishing capacity of 5 .

\begin{tabular}{|c|cc|cc|cc|cc|cc|}
\hline$k$ & MxW & AvW & MxQ & AvQ & MnC & AvC & MxB & AvB & AvP & HfP \\
\hline 0.00 & 65 & 58.19 & 8 & 0.83 & 41 & 51.57 & 24 & 5.78 & 241.00 & 0.00 \\
\hline
\end{tabular}

Figure 2 shows (for the optimal schedule) the number of stills assembled, in the queue, in the charging area, in the buffer and the total number of stills in use for each period during the week. The nonzero values for the queue illustrate that stills are assembled in advance of entering the charging area. The figure shows that the charging area is full for much of the week except on Monday morning and Friday afternoon. The minimum number of stills undergoing charging is 41 on Monday and Friday. The maximum size of the buffer in front of the finishing area is 24 stills on Monday morning and is reduced to zero several times during the week. During some periods the resource limits are reached for the total number of the stills, the charging capacity and the finishing capacity. 


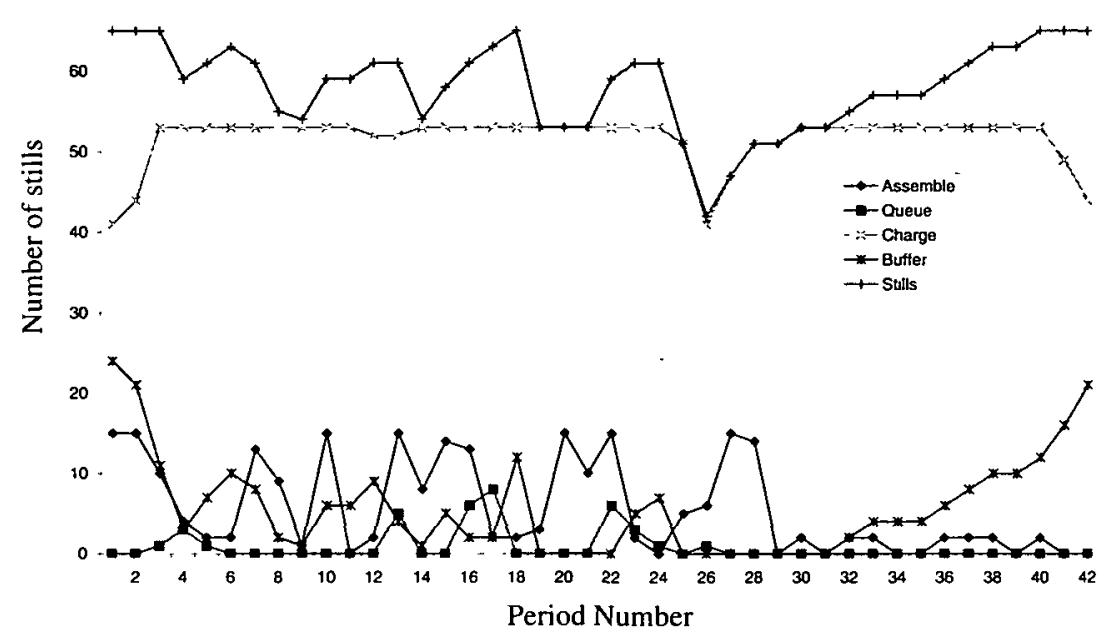

FIGURE 2. Planning model base configuration results showing the number of stills assembled, in the queue, in the charging area, in the buffer and the total number of stills in use for each period during the week.

A comparison between the results above and the first row of Table 3 shows that there is very little discrepancy between the observed statistics for the simulation results and the values we would expect as a result of executing the optimal off-line schedule. Thus we can be confident that there is a high degree of fidelity between the simulation model and the mathematical formulation of the problem.

The most interesting results are gained when we simulate charge-duration disruptions. To do this, as each job enters the charging area its processing time is modified by a normal distribution as described in Section 2 . This perturbation process is used when testing each of the scheduling strategies.

Table 3 gives the performance measures for the optimal off-line schedule as the percentage of charge-duration variability increases. It can be seen that as the variability increases the work in progress remains constant. All of the stills are busy all the time and jobs are released into the model as a still becomes available from the finishing area. As the variability increases there is a steady decline in the average production while the other measures show little variation.

Figure 3 shows the weekly production and the spaces used for 20 weeks after the 2 week warm up period when the disruption ratio is $k=0.02$. It can be seen that production oscillates between finishing 241 stills and about 160 stills. We postulate that the high production corresponds to releasing the stills on schedule while the low production occurs when the release of stills "gets off schedule". It is important that 
TABLE 3. Results for the planning schedule showing the outputs for variation in the disruption ratio with 53 spaces, 65 stills and 5 stills finishing capacity per line.

\begin{tabular}{|r|rr|rr|rr|rr|rr|}
\hline$k$ & MxW & AvW & MxQ & AvQ & MnC & AvC & MxB & AvB & AvP & HfP \\
\hline 0.00 & 65 & 60.40 & 12 & 1.40 & 25 & 50.71 & 39 & 6.79 & 241.00 & 0.00 \\
0.01 & 65 & 60.53 & 12 & 1.61 & 25 & 50.96 & 38 & 6.44 & 241.00 & insuf \\
0.02 & 65 & 64.99 & 12 & 4.11 & 0 & 46.68 & 65 & 12.74 & 219.66 & 0.64 \\
0.03 & 65 & 64.99 & 12 & 3.94 & 0 & 45.71 & 65 & 13.94 & 214.02 & insuf \\
0.04 & 65 & 65.00 & 12 & 3.90 & 0 & 45.22 & 65 & 14.51 & 210.67 & 0.19 \\
0.05 & 65 & 65.00 & 12 & 3.91 & 0 & 44.77 & 65 & 14.97 & 207.58 & insuf \\
0.06 & 65 & 65.00 & 12 & 3.91 & 0 & 44.72 & 65 & 15.04 & 206.37 & insuf \\
0.07 & 65 & 65.00 & 12 & 3.95 & 0 & 44.44 & 65 & 15.26 & 204.04 & insuf \\
0.08 & 65 & 65.00 & 12 & 3.96 & 0 & 44.14 & 65 & 15.57 & 201.67 & 0.51 \\
0.09 & 65 & 65.00 & 12 & 3.91 & 0 & 44.15 & 65 & 15.66 & 200.80 & insuf \\
\hline
\end{tabular}

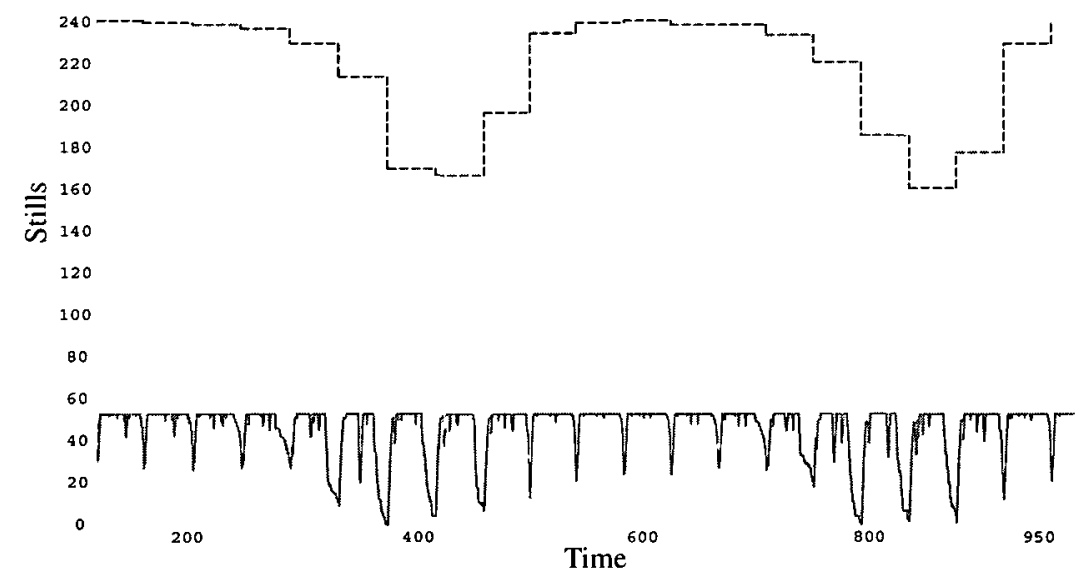

FIGURE 3. Weekly production and space usage as a function of time for the MIP planning schedule with $k=0.02$.

charging of stills occurs on the weekend and it can be seen that the charging-bay usage is close to zero when production is low. Average production is about 220 rather than the demand of 241 and the weekly deficiency of about 20 units gives a periodicity of about 12 weeks to the production plot. Figure 4 shows the weekly production for each of the three scheduling strategies.

For the SPT strategy we rank the products by non-decreasing charge time (that is, the reverse order to that in Table 1 ) and release 241 stills with the correct product mix for each of the 21 products. Table 4 shows that as the disruption percentage increases the number of stills finished decreases, the work in progress remains almost constant, the transit time of jobs increases, the charging area becomes more congested and the buffer decreases. The maximum number of 65 stills are used and the average number 
TABLE 4. Results for the shortest processing time schedule showing the outputs for variation in the disruption ratio with 53 spaces, 65 stills and 5 stills finishing capacity per line.

\begin{tabular}{|r|rr|rr|rr|rr|rr|}
\hline$k$ & MxW & AvW & MxQ & AvQ & MnC & AvC & MxB & AvB & AvP & HfP \\
\hline 0.00 & 65 & 65.00 & 12 & 3.02 & 0 & 40.56 & 64 & 20.08 & 192.75 & insuf \\
0.01 & 65 & 65.00 & 12 & 3.08 & 0 & 40.77 & 65 & 19.87 & 192.75 & insuf \\
0.02 & 65 & 65.00 & 12 & 3.14 & 0 & 40.97 & 65 & 19.61 & 192.75 & insuf \\
0.03 & 65 & 65.00 & 12 & 3.22 & 0 & 41.17 & 65 & 19.32 & 192.83 & insuf \\
0.04 & 65 & 65.00 & 12 & 3.29 & 0 & 41.37 & 65 & 19.04 & 192.75 & insuf \\
0.05 & 65 & 65.00 & 12 & 3.27 & 0 & 41.58 & 65 & 18.88 & 192.79 & insuf \\
0.06 & 65 & 65.00 & 12 & 3.24 & 0 & 41.77 & 65 & 18.74 & 192.73 & insuf \\
0.07 & 65 & 65.00 & 12 & 3.28 & 0 & 41.88 & 65 & 18.59 & 192.31 & insuf \\
0.08 & 65 & 65.00 & 12 & 3.33 & 0 & 41.92 & 65 & 18.48 & 191.55 & 0.61 \\
0.09 & 65 & 65.00 & 12 & 3.35 & 0 & 42.03 & 65 & 18.34 & 191.20 & 0.62 \\
\hline
\end{tabular}

of stills busy increases as the disruption percentage increases. The minimum number on charge increases and the average number charging increases. The number in the buffer does not change significantly as the disruption percentage increases. If anything the maximum number and the average number in the buffer decline as the production declines and less jobs are released into the system.

Figure 4 shows the SPT weekly production and the spaces used for 20 weeks after the 2 week warm up period when the disruption ratio is $k=0.02$. It can be seen that production oscillates between finishing about 220 stills and about 140 stills. Average production is about 193 rather than the demand of 241 and the weekly deficiency of about 50 units gives a periodicity of about 5 weeks to the production plot.

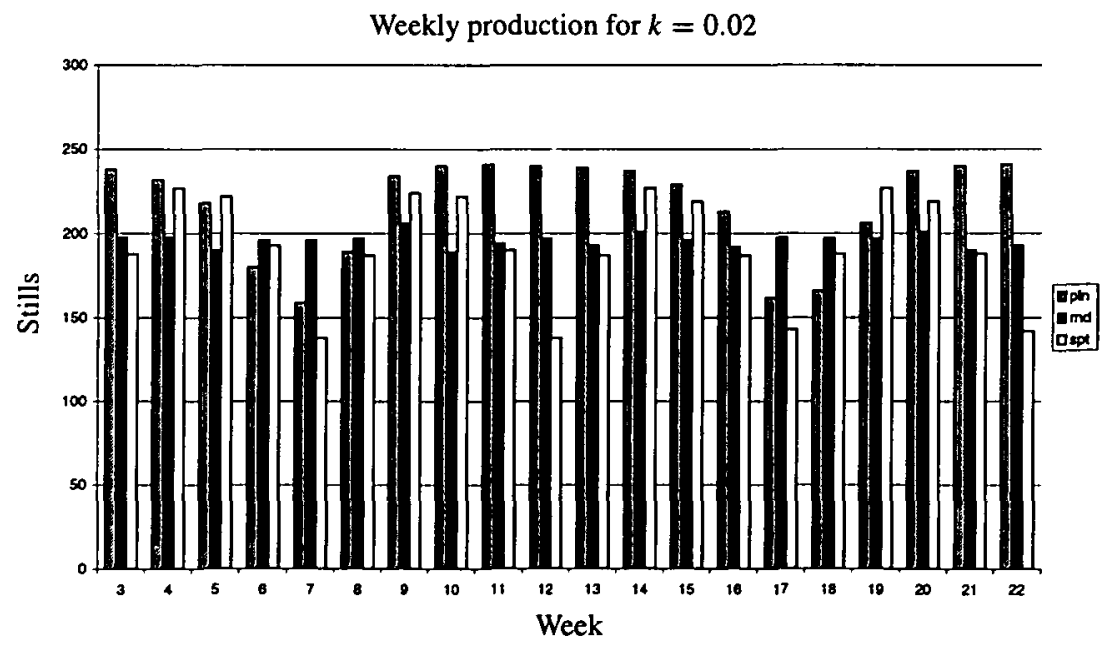

FIGURE 4. Weekly production for the MIP, SPT and RND schedules with $k=0.02$ as a function of time over 20 weeks. 
TABLE 5. Results for the random schedule showing the outputs for variation in the disruption percentage.

\begin{tabular}{|r|rr|rr|rr|rr|rr|}
\hline$k$ & MxW & AvW & MxQ & AvQ & MnC & AvC & MxB & AvB & AvP & HfP \\
\hline 0.00 & 65 & 65.00 & 12 & 3.96 & 1 & 41.76 & 64 & 17.90 & 198.48 & 0.06 \\
0.01 & 65 & 65.00 & 12 & 4.00 & 2 & 41.75 & 63 & 17.88 & 197.42 & insuf \\
0.02 & 65 & 65.00 & 12 & 4.01 & 1 & 41.79 & 64 & 17.85 & 196.66 & insuf \\
0.03 & 65 & 65.00 & 12 & 4.04 & 2 & 41.82 & 63 & 17.81 & 195.83 & 0.02 \\
0.04 & 65 & 65.00 & 12 & 4.06 & 1 & 41.95 & 63 & 17.65 & 195.46 & insuf \\
0.05 & 65 & 65.00 & 12 & 4.09 & 2 & 41.96 & 63 & 17.62 & 194.58 & insuf \\
0.06 & 65 & 65.00 & 12 & 4.11 & 2 & 41.98 & 63 & 17.57 & 193.71 & 0.15 \\
0.07 & 65 & 65.00 & 12 & 4.22 & 2 & 41.99 & 63 & 17.48 & 192.81 & insuf \\
0.08 & 65 & 65.00 & 12 & 4.13 & 3 & 42.19 & 61 & 17.36 & 192.80 & insuf \\
0.09 & 65 & 65.00 & 12 & 4.18 & 2 & 42.21 & 63 & 17.29 & 191.98 & 0.15 \\
\hline
\end{tabular}

The throughput under the RND strategy is resilient to disruptions but nevertheless is low overall. Table 5 gives the performance measures as the variability increases. It can be seen that as the variability increases the queue in front of the charger increases, the charge area fills, the buffer in front of the finishing area decreases and the production falls. As the variability increases the system behaves in a similar manner to the MIP strategy. The main difference is that fewer stills are finished but the reduction in production is not as fast as in the planning model which starts from a higher throughput value.

Figure 4 shows the weekly production and the spaces used for 20 weeks after the 2 week warm up period when the disruption ratio is $k=0.02$. It can be seen that production oscillates between finishing about 200 stills and about 190 stills. Average production is about 196 rather than the demand of 241 and the weekly deficiency of about 45 units gives a periodicity of about 6 weeks to the production plot. The RND schedule does better than the SPT schedule because, when job release is delayed, the schedule maintains a range of processing times.

3.1. Comparison of strategies when constraints are tight We have compared the relative efficiency of production for the three strategies. Figure 5 shows the average weekly production for each of the three strategies as the disruption ratio increases. The figure indicates that the optimized schedule from the MIP model continues to outperform the other strategies as the variation in the processing times increases.

Also we compared the robustness of production for the three strategies. Figure 6 shows the robustness for each of the three strategies as the disruption ratio increases. The figure indicates that the optimized schedule from the MIP model is the mo't robust strategy for small values of the disruption ratio but the random strategy is more robust than the other strategies as the variation in the processing times increases. We reason that the random strategy is insensitive to disruptions because generally there are jobs of all durations in the charging area. 


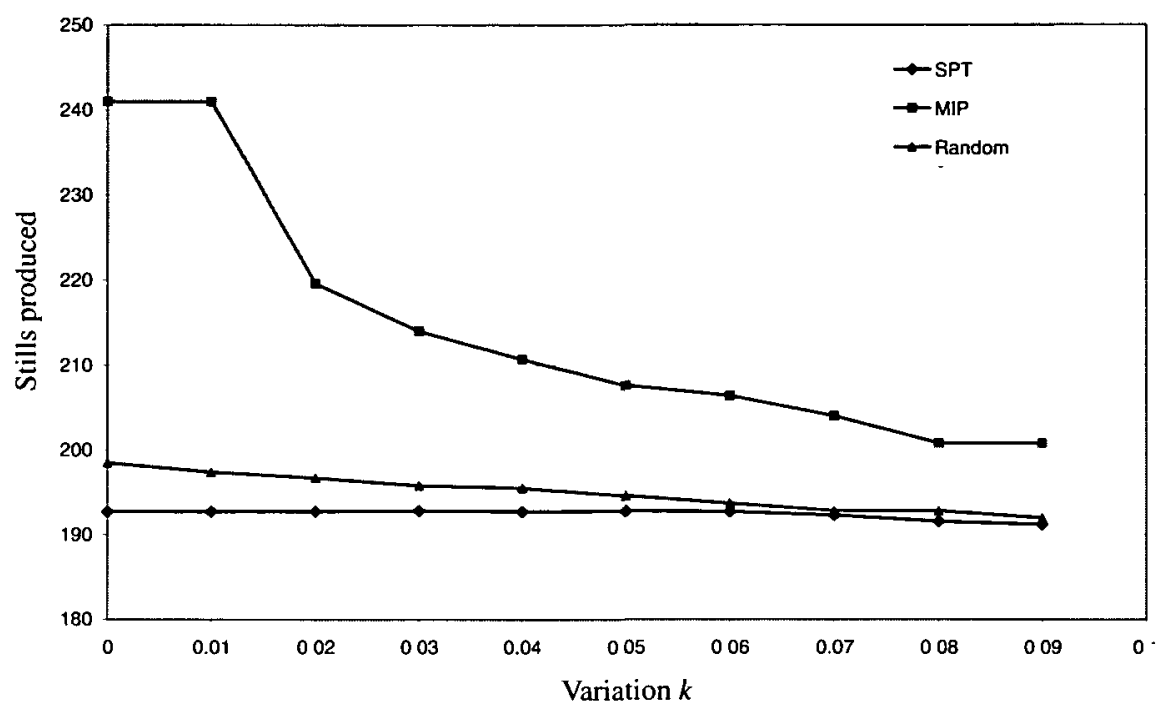

FIGURE 5. Comparison of the performance of the three strategies for 53 spaces and 65 stills showing average weekly production as the disruption ratio $k$ increases.

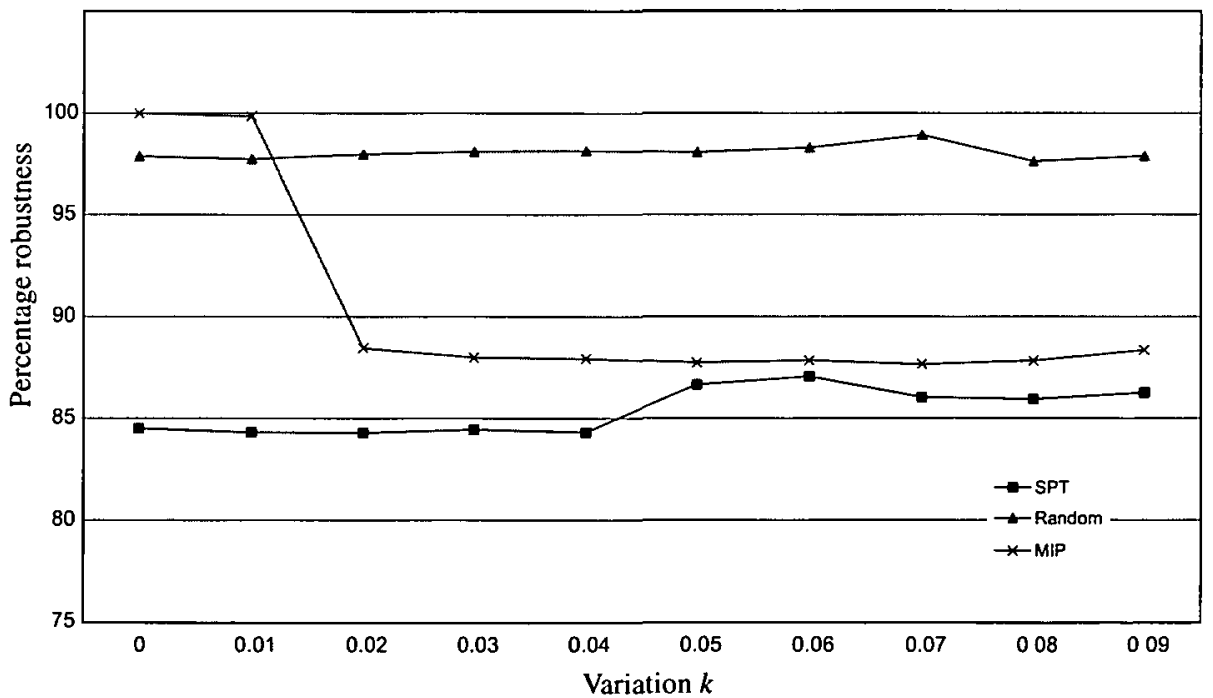

FIGURE 6. Comparison of the robustness of the three strategies for 53 spaces and 65 stills showing robustness as the disruption ratio $k$ increases.

3.2. Comparison of strategies when the constraints are loose We compared the relative efficiency of production for the 3 schedules when the resources available were increased to 55 spaces and 73 stills. The previously-optimal off-line schedule was 


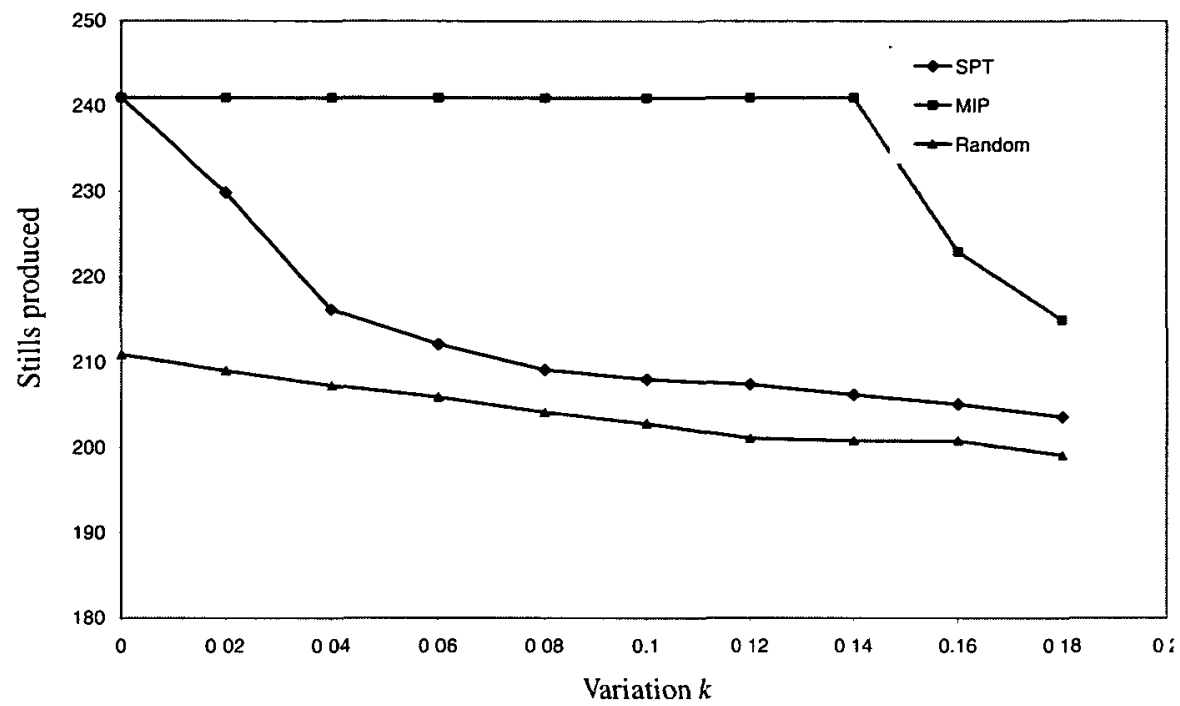

FIGURE 7. Comparison of the performance of the three strategies for 55 spaces and 73 stills showing average weekly production as the disruption ratio $k$ increases.

not re-optimized for the increase in resources. Figure 7 shows the average weekly production for each of the three schedules as the disruption ratio increases. The figure indicates that the previously-optimal off-line schedule outperforms the other schedules for all values of the disruption ratio. The SPT schedule is better than the RND schedule for all values of the disruption ratio. In the absence of disruptions the MIP and SPT schedule give the maximum production of 241 units.

Again we compared the robustness of production for the three strategies when there are 55 spaces and 73 stills available. Figure 8 shows the robustness for each of the three strategies as the disruption ratio increases. The figure indicates that the optimized schedule from the MIP model is the most robust strategy for values of the disruption ratio up to 0.14 but the random strategy is more robust than the other strategies as the variation in the processing times increases. Again we reason that the random strategy is insensitive to disruptions because generally there are jobs of all durations in the charging area.

\section{Conclusions}

In this paper we have studied a multi-product battery charging and finishing facility that produces completed and charged batteries. Batteries are produced in a weekly cycle in our model of such a facility and this is a reasonable approximation to current practice in a major Australian plant that has been the subject of a previous paper. Over 


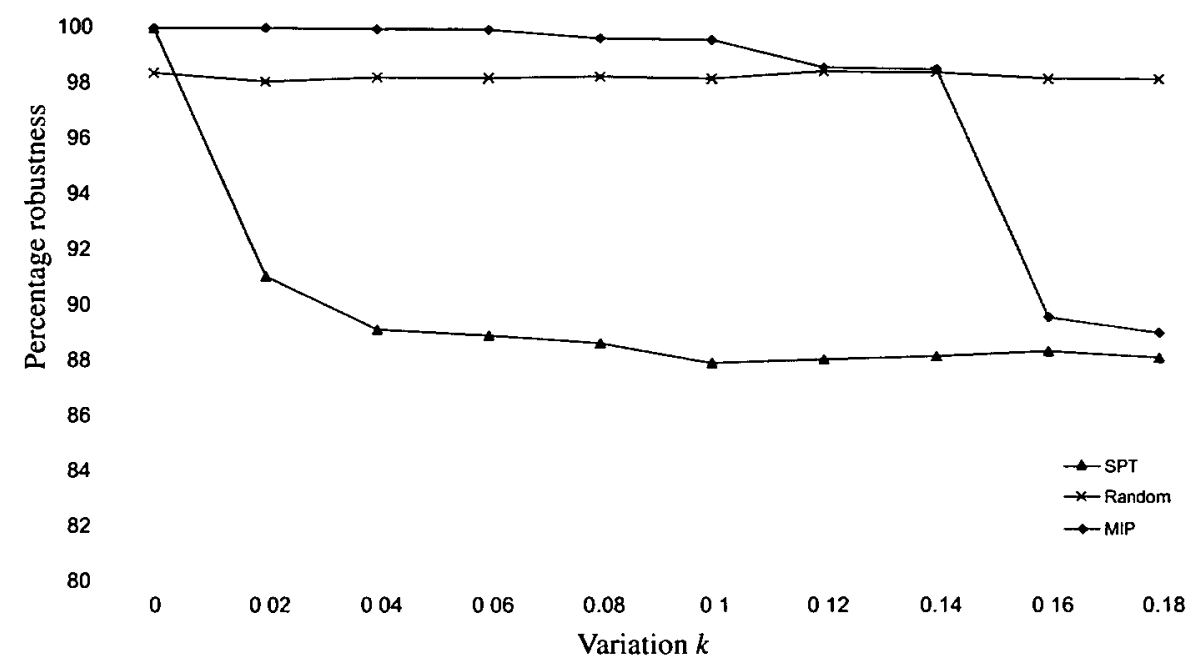

FIGURE 8. Comparison of the robustness of the three strategies for 55 spaces and 73 stills showing robustness as the disruption ratio $k$ increases.

the weekly cycle the facility must achieve a pre-specified ratio of production between products (that is, a certain product mix), and an instance of the cyclic scheduling problem is defined by this ratio, the resource constraints of the facility, the operating intervals of the charging and finishing areas, and the battery charge times for each product. There are three potentially constraining resources in the scheduling problem: the total number of stills upon which groups of batteries (of the same product) are placed, the number of battery charging bays, and the throughput rate of the finishing area.

In our experiments we have simulated (by way of discrete event simulation) the performance of the facility under two distinct scheduling strategies: off-line optimization, and on-line dispatching by way of a simple priority rule. Our goal has been to assess the merits of each strategy through reference to the observed performance of the simulated system under varying conditions. The performance of the system has been characterized in terms of the average number of stills of batteries produced during weekly cycles where the product-mix must be maintained. We have varied the simulation conditions by introducing random perturbing effects that extend battery charging times.

The simulations have shown, under various degrees of perturbation and in a tightlyconstrained system, that following an optimal off-line cyclic schedule is a significantly better strategy than adopting on-line shortest-processing-time dispatching. When the resource constraints provide for more flexibility in the system the off-line and dispatch- 
ing strategies are equally good for minor perturbations, but the relative performance of dispatching deteriorates as the random effects are amplified. These observations are highly relevant for the in-practice operation of such facilities because they dispel the concerns expressed to us by facility managers in relation to the robustness of optimal off-line schedules under the significant charging delays that can occur due to ambient air flow, humidity and temperature. Indeed, an optimal off-line schedule is highly robust in this context, because (a) the executed schedule is invariant to disruptions, and (b) the throughput of the facility remains acceptable.

From the simulation results we can also propose that, at the expense of scheduling robustness as assessed relative to schedule invariance, there may exist sophisticated online approaches that can achieve higher facility throughput than either of the strategies investigated in this paper.

\section{References}

[1] Arena Software, ht tp://www. arenasimulation. com, 2004.

[2] S. Dunstall and R. G. J. Mills, "Scheduling the charging of batteries", in Proc. Mathematics-inIndustry Study Group, (2002), 87-105.

[3] H. S. Gan, "Robust scheduling"; Ph. D. Thesis, University of Melbourne, Australia, 2004.

[4] S. J. Honkomp, L. Mockus and G. V. Reklaitis, "A framework for schedule evaluation with processing uncertainty", Computers Chem. Eng. 23 (1999) 595-609.

[5] E. L. Lawler, J. K. Lenstra, A. H. G. Rinnooy Kan and D. B. Shmoys, "Sequencing and scheduling: algorithms and complexity", in Handbooks in Operations Research and Management Science (eds. S. C. Graves, A. H. G. Rinnooy Kan and P. H. Zipkin), Volume 4, (Elsevier, 1993).

[6] K. C. Liu, "Dispatching rules for stochastic finite capacity scheduling", Computers Ind. Engng 35 (1995) 113-116.

[7] D. J. Mignon, S. J. Honkomp and G.V. Reklaitis, "A framework for investigating schedule robustness under uncertainty", Computers Chem. Eng. 19 Suppl. (1995) S615-S620.

[8] D. M. Panton and N. Beaumont, "Optimisation of work flow", in Proc. Mathematics-in-Industry Study Group, (1997), 31-51.

[9] A. M. Turnquist, University/industry cooperation on an integrated production planning software system, (Institute of Electrical and Electronic Engineers, 1991) 456-459.

[10] G. E. Vieira, J. W. Herrmann and E. Lin, "Rescheduling manufacturing systems: a framework of strategies, policies and methods", J. Scheduling 6 (2003) 39-62. 